\title{
Highly-Accelerated Real-Time Cine MRI using compressed sensing and parallel imaging
}

\author{
Li Feng, Ricardo Otazo, Monvadi B Srichai, Ruth P Lim, Daniel K Sodickson, Daniel Kim \\ From 2011 SCMR/Euro CMR Joint Scientific Sessions \\ Nice, France. 3-6 February 2011
}

\section{Purpose}

To develop and evaluate highly-accelerated real-time cine MRI using compressed sensing and parallel imaging.

\section{Introduction}

Breath-hold cine MRI with balanced steady-steady free precession (b-SSFP) may yield non diagnostic image quality in patients with impaired breath-hold capacity and/or arrhythmias. In such patients, it may be necessary to perform real-time cine MRI. Currently, dynamic parallel imaging methods, such as TSENSE [1] and TGRAPPA [2], can be used to achieve only moderate acceleration rates $(R)$ of 2-3 using standard body and spine coil arrays. We propose the application of a recently developed joint acceleration technique (CS-PI) [3] that combines compressed sensing [4] and parallel

imaging for highly-accelerated, real-time cine MRI with clinically acceptable spatiotemporal resolution.

\section{Methods}

Real-time cine MRI pulse sequences with b-SSFP readouts and TGRAPPA and CS-PI accelerations with $\mathrm{R}=4$ and $\mathrm{R}=8$ were implemented on $3 \mathrm{~T}$ whole-body MRI scanners (Siemens; Tim-Trio \& Verio) equipped with standard body and spine coil arrays (12 elements total). The relevant imaging parameters include: $F O V=320 \mathrm{~mm}$ $\mathrm{x} 320 \mathrm{~mm}$, acquisition matrix size $=128 \times 128, \mathrm{TE} /$ $\mathrm{TR}=1.37 / 2.7 \mathrm{~ms}$, receiver bandwidth $=1184 \mathrm{~Hz} /$ pixel, and flip angle $=40^{\circ}$. The temporal resolutions were 86.4, and $43.2 \mathrm{~ms}$ for $\mathrm{R}=4$ and 8 , respectively. Seven patients (mean age $=41.5 \pm 20.7$ years) undergoing clinical CMR were imaged in mid-ventricular short-axis and long-axis planes, following completion of the clinical examination

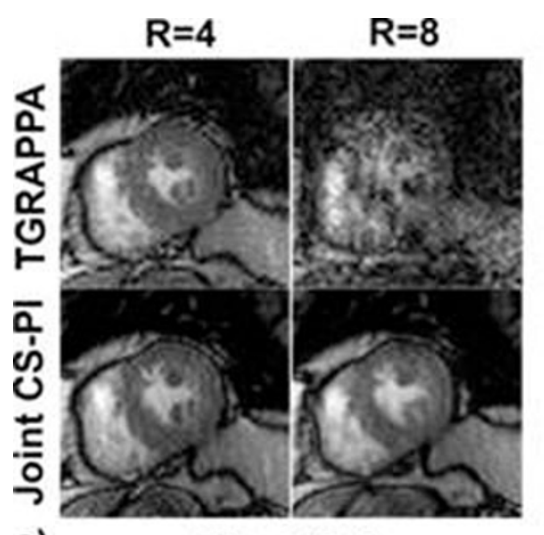

a)

Short-Axis

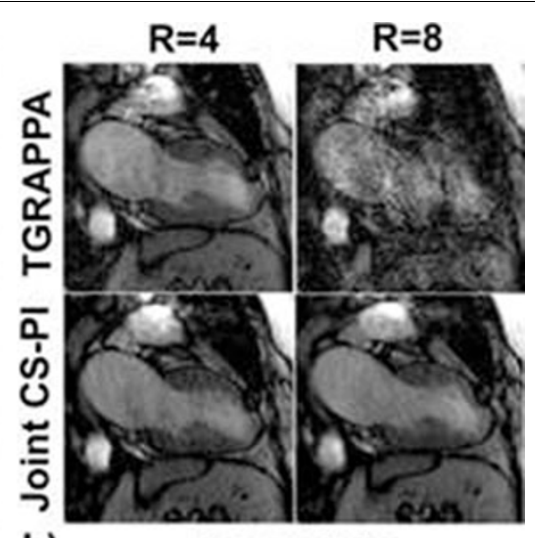

b)

Long-Axis

Figure 1 a) Short-axis view and b) long-axis view. For each view: (top row) TGRAPPA; (bottom row) joint CS-PI; (left column) R=4; (right column) $R=8$.

New York University School of Medicine, New York, NY, USA

(c) 2011 Feng et al; licensee BioMed Central Ltd. This is an open access article distributed under the terms of the Creative Commons 


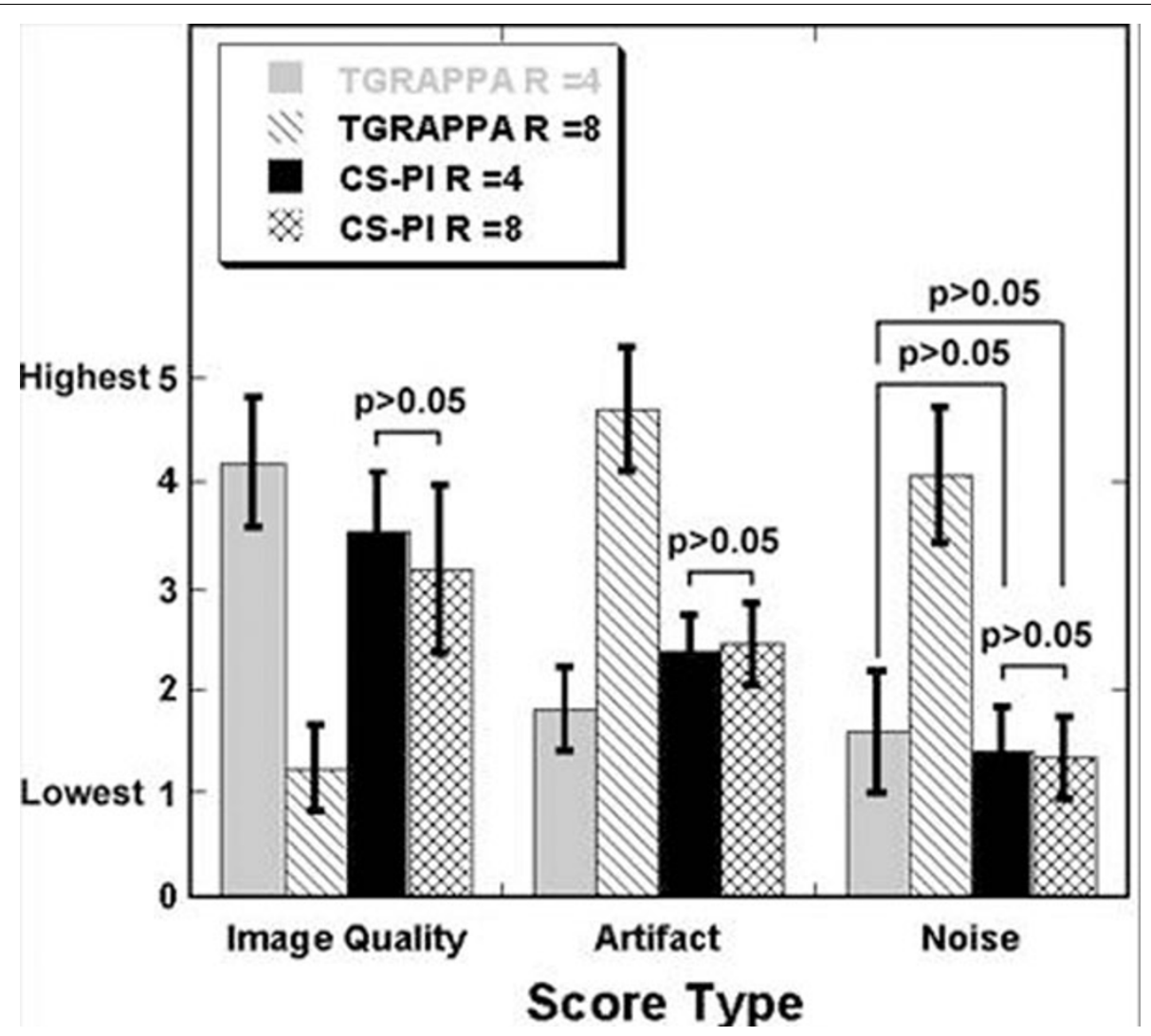

Figure 2 Plots of mean scores: (left) image quality, (middle) artifact, and (right) noise. Pairs with no significant difference are noted with $p>0.05$.

using free breathing and electrocardiogram gating. The cine data sets were randomized and blinded for qualitative evaluation (image quality, artifact, noise; $1-5$; lowest-highest) by a cardiologist and a radiologist. Statistical analysis was performed to compare the mean scores between the 4 groups (TGRAPPA-R4, TGRAPPA-R8, CS-PI-R4, CS-PI-R8) and between each pair of groups.

\section{Results}

Figure 1 shows images of end-systolic frames in midventricular short-axis and 2-chamber views. According to the Kruskal-Wallis test, the 4 groups were significantly different (p0.05): image quality, CS-PI-R4 vs. CS-PI-R8; artifact, CS-PI-R4 vs. CS-PI-R8; noise, TGRAPPA-R4 vs. CS-PI-R8, TGRAPPA-R4 vs. CS-PIR4, CS-PI-R4 vs. CS-PI-R8. These preliminary results suggest that TGRAPPA can yield robust results at $R=4$, whereas CS-PI can yield robust results up to $R=8$.

\section{Discussion}

This study demonstrates the feasibility of performing highly-accelerated real-time cine MRI using a joint CSPI technique. An 8-fold accelerated real-time cine MRI protocol can achieve spatial resolution of $2.5 \mathrm{~mm} \mathrm{x}$ $2.5 \mathrm{~mm}$ and temporal resolution of $43.2 \mathrm{~ms}$, with adequate image quality. This accelerated protocol may be useful for debilitated patients with reduced breathhold capacity and/or arrhythmias for rapid left ventricular functional evaluation.

Published: 2 February 2011

\section{References}

1. Kellman P, et al: MRM 2001

2. Breuer FA, et al: MRM 2005.

3. Otazo R: MRM 2010.

4. Lustig M, et al: MRM 2007.

doi:10.1186/1532-429X-13-S1-P25

Cite this article as: Feng et al:: Highly-Accelerated Real-Time Cine MRI using compressed sensing and parallel imaging. Journal of Cardiovascular Magnetic Resonance 2011 13(Suppl 1):P25. 